The successful cloning of the gene has important consequences, as a PCR can now be employed to establish the diagnosis of FMF in suspected patients. ${ }^{3}$ Prophylactic colchicine therapy may be offered to patients with FMF, as the attacks under colchicine therapy are milder, of shorter duration, and may prevent renal failure. ${ }^{1}$

1 Matzner Y. Biologic and clinical advances in familial Mediterranean fever. Crit Rev Oncol Hematol 1995;18:197-205.

2 Matzner Y, Brzezinski A. C5a-inhibitor deficiency in peritoneal fluids from patients with familial Mediterranean fever. N Engl f Med 1984;311:287-90.

3 The International FMF Consortium. Ancient missense mutations in a new member of the RoRet gene family are

\section{Final diagnosis}

Familial Mediterranean fever.

Keywords: familial Mediterranean fever; abdominal pain

likely to cause familial Mediterranean fever. Cell 1997;90: 797-807.

4 The French FMF Consortium. A candidate gene for familial Mediterranean fever. Nat Genet 1997;17:25-31.

5 Goldfinger SE. Colchicine for familial Mediterranean fever. N Engl f Med 1972;287:1302.

\title{
Small bowel obstruction in a young adult
}

\author{
D W Harkin, G Blake
}

A 21-year-old man presented to hospital with a 3-day history of increasing vomiting, abdominal pain and distension, and constipation. On questioning, he gave a 3-month history of poor appetite, intermittent abdominal cramps, intermittent diarrhoea and weight loss of approximately 13 $\mathrm{kg}$. He had no relevant medical or family history, and had no previous surgery. He was apyrexic clinically anaemic, with a centrally distended abdomen, but no abdominal scars or external hernia. Bowel sounds were obstructive but there were no obvious signs of peritonism. Positive blood results showed a microcytic hypochromic anaemia (haemoglobin $7.9 \mathrm{~g} / \mathrm{dl}$ ) and a low serum albumin (corrected albumin $26 \mathrm{~g} / \mathrm{l}$ ), his inflammatory markers were also grossly elevated (C-reactive protein (CRP) $112 \mathrm{IU} / 1$; erythrocyte sedimentation rate 77$)$. Erect and supine abdominal X-rays are shown in figures 1 and 2 .

\section{Department of Surgery, Daisy Hill Hospital, Newry, Northern Ireland D W Harkin G Blake}

Correspondence to Mr DW Harkin, Specialist Registrar General Surgery, 37 Wynchurch Terrace, Rosetta, Belfast BT6 OHP, Northern Ireland

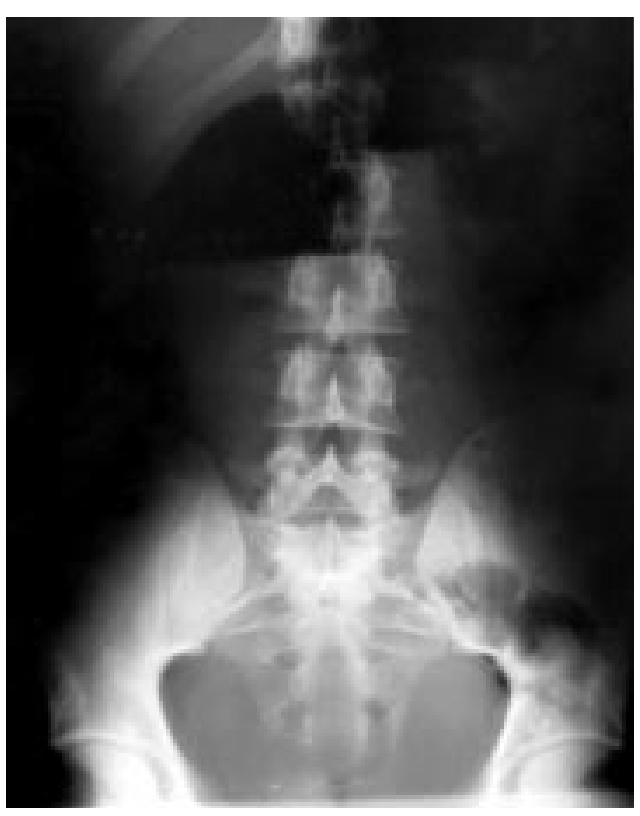

Figure 1 Erect abdominal X-ray

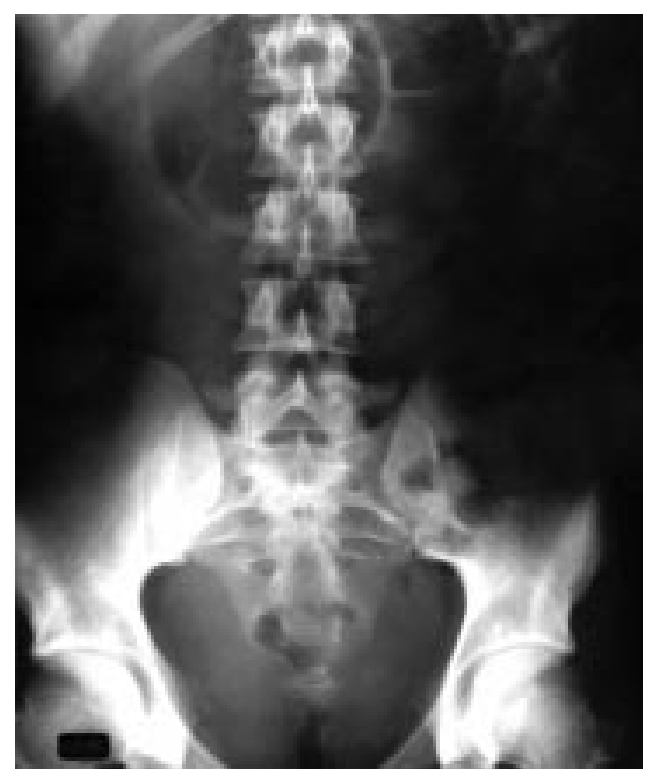

Figure 2 Supine abdominal X-ray

\section{Questions}

1 What do the abdominal X-rays show (figures 1 and 2)?

2 What is the differential diagnosis?

3 What is the most appropriate treatment? 


\section{Answers}

QUESTION 1

The erect abdominal X-ray (figure 1) shows a large air-filled viscus in the upper abdomen with two long fluid levels. The supine abdominal $\mathrm{X}$-ray (figure 2) shows several grossly dilated small bowel loops in the central upper abdomen. No obvious free intra-peritoneal gas is seen outlining the ligamentum teres, major viscus, paracolic gutters, or between adjacent bowel loops (Riggler's sign) on either X-ray. An erect chest film also showed no subdiapragmatic air.

\section{QUESTION 2}

The differential diagnosis is small bowel obstruction (adhesions, hernia, tumour, inflammatory stricture, foreign body, gallstone, congenital bands or stenosis, intussusception, meconium ileus or meconium ileus equivalent, mid-gut volvulus), large bowel obstruction (tumour, volvulus, inflammatory stricture), or gastric dilation (acute, outlet obstruction, volvulus).

The distribution of the distended bowel loop in the central upper abdomen, the presence of valvulae conniventes, and the absence of gaseous distension of the caecum implies small bowel obstruction. The size of the distended bowel loop may imply an acute on chronic obstruction.

\section{QUESTION 3}

He underwent a surgical laparotomy which revealed an obstructed and grossly dilated 25 $\mathrm{cm}$ segment of mid small bowel twisted $360^{\circ}$ clockwise about its mesentery (figure 3 ). The segment of small bowel was grossly dilated with diameter in excess of $10 \mathrm{~cm}$, but was not acutely ischaemic, and there were many large reactive lymph nodes in its draining mesentery. There was no evidence of malrotation or situsinversus, and there were no abnormal fibrous or Ladd's bands. We performed a wide segmental resection and side-to-side anastamosis of his small bowel. He made an uncomplicated post-operative recovery.

Histopathology showed a gross specimen of $25 \mathrm{~cm}$ of dilated small bowel, diameter in excess of $10 \mathrm{~cm}$, with four large ulcerated areas on the mucosal surface and unremarkable mucosa in between. The mesentery also contained two large reactive lymph nodes. On histological examination the areas of mucosal ulceration contained fissure ulcers, transmural chronic ulceration, lymphoid follicle forma-

Figure 3 Operative field at laparotomy, showing a grossly dilated segment of small bowel. the base of the dilated segment is seen to be twisted $360^{\circ}$ in a clockwise direction, forming a closed loop obstruction tion, and submucosal fibrosis. Areas of normal mucosa separated the involved segments. Despite the absence of granulomata, histopathological features were consistent with Crohn's disease.

Our patient is presently under review on sulphasalazine treatment, and has had no major disease flare-ups or symptoms one year post operation.

\section{Discussion}

Crohn's disease is a chronic, transmural, inflammatory, disease of the intestinal tract most frequently involving the terminal ileum and colon. The disease can affect any part of the gastrointestinal tract from lips to anus, and also can manifest itself as various systemic complications such as finger clubbing, large joint arthritis, erythema nodosum, iritis, pyoderma gangrenosum, episcleritis, uveitis and conjunctivitis, sclerosing cholangitis and bile duct carcinoma, although liver problems are much more common in ulcerative colitis. ${ }^{12}$ There is a wide geographical variation in the incidence of Crohn's disease, and although the aetiology is unknown, there does appear to be some genetic susceptibility as there is a 30 -fold increase in sibling incidence compared to the general population. The most common symptoms of small bowel Crohn's disease are diarrhoea (90\%), abdominal pains (55\%), anorexia, nausea and weight loss $(22 \%) .{ }^{12}$ Our patient exhibited all these symptoms, and also showed typical nutritional disturbance of anaemia (iron, folate or vitamin B12 deficiency), hypoalbuminaemia and weight loss. Acute phase reactants are also often raised in active disease and our patient's CRP was grossly elevated. Small bowel stenosis is common in Crohn's disease, at time of diagnosis a bowel stenosis was documented in $37 \%$ in one series. ${ }^{3}$ However, acute first presentation of Crohn's disease with small bowel segmental volvulus has not previously been reported. Closed loop obstruction such as this can also lead to accelerated mucosal permeability changes with bacterial translocation and portal endotoxaemia which may lead to multiple organ dysfunction syndrome. Also grossly distended bowel is at risk of ischaemic necrosis and/or perforation. ${ }^{3}$ Therefore, early diagnosis and expedient treatment is a priority to prevent the development of these complications. Small bowel volvulus (midgut volvulus) is in itself rare and usually presents in childhood, being due to an unusually narrow based mesentery to the small bowel caused by malrotation of the bowel and persistent embryological peritoneal bands (Ladd's bands). In the absence of this embryological abnormality, volvulus of small bowel is extremely rare. However, in our case, there was no malrotation and rather the volvulus occurred by twisting of a grossly dilated loop of small bowel about an area of inflammatory stenosis of the small bowel. With short segment stenosis due to Crohn's disease, the conservative procedure of stricturoplasty has been very effective with a low associated morbidity and mortality. ${ }^{4}$ In our patient however, due to the 
extent of the disease in the involved segment and the lack of a prior histological diagnosis, a segmental resection was felt to be appropriate. Clinicians should be aware of this rare presentation, demonstrated on abdominal X-ray, which required quick diagnosis and urgent treatment. This report adds to the known literature on both Crohn's disease and small bowel volvulus.

\section{Final diagnosis}

Crohn's disease presenting as a segmental small bowel volvulus.

Keywords: Crohn's disease; volvulus; small bowel obstruction

\section{Learning points}

- Crohn's disease can present in a variety of ways and mimic many other disease processes. It should be part of every clinician's differential diagnosis of acute or chronic abdominal pain

- a closed loop bowel obstruction is a surgical emergency which requires rapid diagnosis and early intervention, if a successful outcome is to be obtained

- true midgut volvulus not only presents in children, but although rare, has been reported in young adults and even geriatrics

- in the absence of congenital abnormalities, a volvulus can occur around any pivotal point; common axes for the volvulus are tumours, adhesive bands, and stenoses
1 Maeda $\mathrm{K}$, Okada $\mathrm{M}$, Yao $\mathrm{T}$, et al. Intestinal and extra-intestinal complications of Crohn's disease: predictors and cumulative probability of complications. $\mathcal{F}$ Gastroenterol and cumulative

2 Grau A, Quintana C, Zuniga A. Crohn's disease: clinical presentation and results of surgical treatment. Rev Med Chile 1993;121:162-6.
3 Makowiec F, Jehle EC, Koveker G, Becker HD, Starlinger $M$. Intestinal stenosis and perforating complications in Crohn's disease. Int f Colorectal Dis 1993;8:197-200.

4 Nivatvongs S. Stricturoplasty for Crohn's disease of small intestine. Present status in Western countries. $\mathcal{F}$ Gastroenterol 1995;30(suppl 8):139-42.

\title{
Atrial flutter in a young man with a highly competitive and stressful occupation
}

\author{
S W Dubrey, A S Kurbaan, S Kaddoura
}

A previously fit 41-year-old man presented in March 1997 with palpitations and shortness of breath. Initial symptoms consisted of paroxysmal palpitations occurring after exercising; however, over the course of 6 months, he became aware that his pulse was consistently irregular and that even mild exertion made him short of breath. The patient was on no medicines. Coffee, tea and caffeine-containing beverages were only consumed in moderation. He was a life-long non-smoker and consumed approximately 5 units of alcohol per week. His occupation was competitive with a mentally stressful environment; he found his performance was helped by the use of a dietary supplement. Examination was unremarkable apart from an irregularity of the arterial pulse. The patient appeared euthyroid and was normotensive (blood pressure $110 / 700 \mathrm{mmHg}$ ) with no signs of heart failure. An electrocardiogram showed atrial flutter, at a rate of 148 beats $/ \mathrm{min}$, with variable block. Haematological and biochemical analyses, including thyroid function were normal as were a chest radiograph and echocardiogram. The patient was anticoagulated with warfarin and commenced on flecainide (200 mg bid) in anticipation of precipitating a pharmacologic cardioversion to sinus rhythm.

Administration of flecainide resulted in a deterioration in symptoms due to increased irregularity of heart rhythm and a reduction in exercise capacity. This was discontinued and an elective DC cardioversion was performed with a return to sinus rhythm at a rate of 68 beats $/ \mathrm{min}$.

\section{Questions}

1 What is the most probable diagnosis?

2 What would be your next investigation?

3 In what cardiovascular circumstance might you have chosen to avoid flecainide because of its pro-arrhythmic potential? 